\title{
Uniqueness, symmetry and full regularity of free boundary in optimization problems with volume constraint
}

\author{
EDUARDO V. TEIXEIRA ${ }^{\dagger}$ \\ Department of Mathematics, Rutgers University, \\ Hill Center-Busch Campus, 110 Frelinghuysen Road, Piscataway, NJ 08854-8019, USA, and \\ Departamento de Matemática, Universidade Federal do Ceará, \\ 60455-760 Fortaleza, CE, Brasil
}

[Received 1 February 2006 and in revised form 27 October 2006]

\begin{abstract}
We study qualitative geometric properties of optimal configurations to a variational problem with free boundary, under suitable assumptions on a fixed boundary. More specifically, we study the problem of minimizing the flow of heat given by $\int_{\partial D} \Gamma\left(u_{\mu}\right) \mathrm{d} \sigma$, where $D$ is a fixed domain and $u$ is the potential of a domain $\Omega \supset \partial D$, with a prescribed volume on $\Omega \backslash D$. Our main goal is to establish uniqueness and symmetry results when $\partial D$ has a certain geometric property. Full regularity of the free boundary is obtained under these symmetry conditions imposed on the fixed boundary.
\end{abstract}

\section{Introduction}

Given a surface $\partial D \subset \mathbb{R}^{n}$ and a positive function $\phi$ defined on it (temperature distribution of the body $D$ ), a classical minimization problem in heat conduction asks for an optimal configuration $\Omega \supset \partial D$ (insulation) that minimizes the loss of heat in a stationary situation, where the amount of the insulating material is prescribed. This situation also models problems in electrostatics, potential flow, fluid mechanics, among others.

Its mathematical description is as follows: For each domain $\Omega$ surrounding $D$, we consider the potential $u$ associated to the configuration $\Omega$, i.e., the solution of

$$
\begin{cases}\Delta u=0 & \text { in } \Omega \backslash D \\ u=\phi & \text { on } \partial D \\ u=0 & \text { on } \partial \Omega\end{cases}
$$

The flow of heat corresponding to the configuration $\Omega$ is given by

$$
J(\Omega):=\int_{\partial D} \Gamma\left(x, u_{\mu}(x)\right) \mathrm{d} \sigma
$$

where $\mu$ is the inward normal vector defined on $\partial D$. The function $\Gamma: \partial D \times \mathbb{R} \rightarrow \mathbb{R}$ is assumed to be convex and increasing in $u_{\mu}$. Important examples are $\Gamma(t)=t$ (classical heat conduction problem), $\Gamma(t)=t^{p}$ (optimal configurations in electrostatics), $\Gamma(x, t)=\max \{t, C(x)\}$ (problems in material sciences), among many others.

\footnotetext{
${ }^{\dagger}$ E-mail: teixeira@math.rutgers.edu
} 
The optimization problem we are concerned with is to minimize $J(\Omega)$ among all configurations $\Omega$ such that $\operatorname{Vol}(\Omega \backslash D)=1$. In other words, the variational problem we are interested in is

$$
\begin{aligned}
& \operatorname{Minimize}\left\{J(u):=\int_{\partial D} \Gamma\left(x, u_{\mu}(x)\right) \mathrm{d} \sigma \mid\right. u: D^{C} \rightarrow \mathbb{R}, u=\phi \text { on } \partial D \\
&\Delta u=0 \text { in }\{u>0\} \text { and } \operatorname{Vol}(\operatorname{supp} u)=1\} .
\end{aligned}
$$

From the mathematical point of view, Problem (1.1) seems too hard to be directly approached. There are two main reasons for such a technical difficulty. The first one is due to the fact that most of the estimates we obtain involve the capacitance of the configuration, $\int_{D^{C}}|\nabla u|^{2} \mathrm{~d} x$. Therefore the suitable space to look for solutions is $H^{1}\left(D^{C}\right)$. However, normal derivative of merely $H^{1}$ functions is not, in principle, well defined. In the linear case, i.e., when $\Gamma(t)=t$, this difficulty can be overcome by obtaining an equivalent functional to minimize. The second and main difficulty of Problem (1.1) is due to the volume constraint on the support of the temperature. Such a constraint is very unstable under limits and perturbation arguments. In [23] the author considers the following penalized version of Problem (1.1):

Definition 1.1 For $\delta>0$ set $D_{\delta}:=\left\{x \in D^{C}: \operatorname{dist}(x, \partial D)<\delta\right\}$. We define

$$
V_{\delta}:=\left\{u \in H^{1}\left(D^{C}\right): u \geqslant 0, \Delta u \geqslant 0, \Delta u=0 \text { in } D_{\delta}, \text { and } u=\phi \text { on } \partial D\right\}
$$

and

$$
V:=\bigcup_{\delta \searrow 0} V_{\delta}
$$

The penalized problem is stated as follows: Let $\varepsilon>0$ be fixed. We consider the function

$$
f_{\varepsilon}:= \begin{cases}1+\frac{1}{\varepsilon}(t-1) & \text { if } t \geqslant 1 \\ 1+\varepsilon(t-1) & \text { otherwise. }\end{cases}
$$

Consider the problem

$$
\operatorname{minimize} J_{\varepsilon}(u):=\int_{\partial D} \Gamma\left(x, u_{\mu}(x)\right) \mathrm{d} \sigma+f_{\varepsilon}(|\{u>0\}|)
$$

over $u \in V$.

Any function in $V$ is smooth close to the boundary of $D$, thus we are allowed to properly compute the normal derivatives of such a function. Notice that there is no volume constraint any more. Philosophically speaking, the idea is the following: we allow any configuration to compete in our optimization problem, regardless of the volume of the support of the temperature; however, the function $f_{\varepsilon}$ will charge a lot for those configurations that have a volume bigger than 1 . We hope that if the "fee" is too high, optimal configurations of Problem (1.2) will choose to have volume 1 rather than pay for the penalization. Indeed, in [23] the following result is shown:

THEOREM 1.2 If $\varepsilon$ is small enough, then any solution to the minimizing problem (1.2) is a solution to our original Problem (1.1) and vice versa. 
Simplified versions of Problem (1.1) have been considered in [5] $(\Gamma(x, t)=t, \phi \equiv 1)$ and [6] $(\Gamma(x, t)=t)$. Existence and regularity properties of an optimal configuration of Problem $(1.1)$ in its full generality were derived in [23]. The main results in [23] are the following:

(1) There always exists at least one optimal configuration $\Omega \supset \partial D$.

(2) The temperature distribution $u: D^{C} \rightarrow \mathbb{R}$ associated to the optimal configuration $\Omega$ is Lipschitz continuous.

(3) The temperature $u$ growths linearly away from the free boundary $\partial \Omega$, i.e., there exists a universal constant $c>0$ such that

$$
c \operatorname{dist}(x, \partial \Omega) \leqslant u(x) \leqslant c^{-1} \operatorname{dist}(x, \partial \Omega) \quad \text { for all } x \in \Omega .
$$

(4) The free boundary has uniform density, i.e., there exists a universal constant $0<c<1$ such that, for each $x_{0} \in \partial \Omega$,

$$
c \leqslant \frac{\left|\partial \Omega \cap B\left(x_{0}, r\right)\right|}{r^{n-1}} \leqslant 1-c,
$$

where $B\left(x_{0}, r\right)$ is the $n$-dimensional ball centered at $x_{0}$ with radius $r$.

(5) The free boundary $\partial \Omega$ is an analytic surface up to an $(n-1)$-Hausdorff negligible set.

In the present paper we turn our attention to qualitative geometric properties of the free boundary, namely symmetry, uniqueness and full regularity. Clearly, either a nonsymmetric temperature distribution or a symmetry breaking of the $x$ dependence upon the nonlinearity $\Gamma$, ruins any hope of having symmetry properties of the fixed boundary $\partial D$ transported to the free boundary $\partial\{u>0\}$. That is why, in the present paper, we will mostly consider the problem:

Problem 1 Let $\gamma>0$ be a positive constant. Minimize

$$
\begin{aligned}
\left\{J(u):=\int_{\partial D} \Gamma\left(u_{\mu}(x)\right) \mathrm{d} \sigma \mid u: D^{C} \rightarrow \mathbb{R}, u\right. & \equiv \gamma \text { on } \partial D, \\
\Delta u & =0 \text { in }\{u>0\} \text { and } \operatorname{Vol}(\operatorname{supp} u)=1\},
\end{aligned}
$$

where $\Gamma$ is convex and increasing.

Regarding full regularity of the free boundary, let us mention that this is one of the most challenging questions in the theory of free boundary regularity problems. By full regularity of the free boundary we mean that $\partial\{u>0\}=\partial_{\text {red }}\{u>0\}$. It was proven in [23] that $\mathcal{H}^{n-1}\left(\partial\{u>0\} \backslash \partial_{\text {red }}\{u>0\}\right)=0$. Therefore, the set of possible singular points of the free boundary is small. Full regularity of the free boundary ensures that actually the singular set is empty. We recall that, for instance in the free boundary problem studied in [7], in two dimensions, the whole free boundary is regular. In a recent paper, Caffarelli, Jerison and Kenig in [10] showed a full regularity result in three dimensions. There is hope to extend this result up to dimension 6 .

Our paper is organized as follows. In Section 2 we study radial symmetry for Problem 1 . We prove that the best way of insulating a sphere is by another sphere. Due to the volume constraint, this implies uniqueness. The strategy here is to initially study the linear case, $\Gamma(t)=t$. In this setting we show that the temperature distribution in the optimal configuration is radial. We use this information to compare the solution $L$ of the linear case with a solution $u_{\Gamma}$ of Problem 1 with the nonlinearity $\Gamma$. In the next section we study the case when $D$ is starlike with respect to a small neighborhood of the 
origin. For that section we restrict ourselves to the linear setting. Our main theorem in that section asserts that if $D$ is starlike, then the solution to Problem/1 is unique. Moreover, the level sets of the temperature $u$ are starlike and the free boundary $\partial\{u>0\}$ is an analytic surface with no singular points. We should point out that our full regularity result is possible because we impose a geometric constraint on the fixed boundary $\partial D$ which we show is transported to the free boundary $\partial\{u>0\}$. Such a geometric property, combined with further information about the behavior of $\nabla u$ on the free boundary, permits us to conclude that singular points cannot exist. The strategy to show that the free boundary inherits the starlikeness property of $\partial D$ is to compare Problem 1 to two auxiliary Bernoulli-type free boundary problems, where perturbation arguments are easier to apply. In the last section, we study the relation between uniqueness and symmetry. For example we show $O(n)$ symmetry under the uniqueness assumption. At the end of the paper, we raise some questions and conjectures as an invitation for the readers to further develop the theory.

\section{Spherical symmetry}

In this section we shall suppose our body $D$ is a ball in $\mathbb{R}^{n}$. With no loss of generality we will assume $D$ is centered at the origin. In some sense, this is an extreme symmetry case. The perfect symmetry of the body makes it natural to expect that one can infer much more information about the free boundary problem 1.3 .

Let us start by a geometric argument to motivate. Let us restrict ourselves to dimension $n \geqslant 3$. Let $u$ be a solution to Problem 1 for $D=B(R)$, and set $\Omega=\operatorname{supp} u$. Let $B\left(r_{1}\right)$ and $B\left(r_{2}\right)$ be the biggest ball inside $\Omega \backslash D$ and the smallest ball outside $\Omega$ respectively. Let $y_{1} \in B\left(r_{1}\right) \cap \partial \Omega$ and $y_{2} \in B\left(r_{2}\right) \cap \partial \Omega$. Define

$$
u_{i}(x)=\frac{\gamma}{R^{2-n}-r_{i}^{2-n}}|x|^{2-n}-\frac{\gamma r_{i}^{2-n}}{R^{2-n}-r_{i}^{2-n}} \quad \text { for } i=1,2 .
$$

Notice that $u_{i}$ is harmonic, $\left.u_{i}\right|_{\mathbb{S}^{n-1}(R)} \equiv \gamma$ and $\left.u_{i}\right|_{\mathbb{S}^{n-1}\left(r_{i}\right)} \equiv 0$. Furthermore,

$$
D_{j} u_{i}(x)=\frac{\gamma(2-n)}{R^{2-n}-r_{i}^{2-n}} x_{j}|x|^{-n} \quad \text { for } i=1,2 \text { and } j=1, \ldots, n .
$$

Thus, if $v$ denotes the outward normal vector on $\partial \Omega$,

$$
\left(u_{i}\right)_{v}\left(y_{i}\right)=\frac{\gamma(2-n)}{R^{2-n}-r_{i}^{2-n}} r_{i}^{1-n} \quad \text { for } i=1,2 .
$$

Now, from the maximum principle, $u_{1} \leqslant u \leqslant u_{2}$, hence, if $y_{1}, y_{2} \in \partial_{\text {red }} \Omega$,

$$
\left(u_{2}\right)_{v}\left(y_{2}\right) \leqslant u_{v}\left(y_{2}\right) \text { and } u_{v}\left(y_{1}\right) \leqslant\left(u_{1}\right)_{v}\left(y_{1}\right) .
$$

On the other hand, from the free boundary condition found in [23], or even in [5], we know that there exists a negative constant $\lambda$ such that

$$
u_{v} \equiv \lambda \quad \text { on } \partial_{\text {red }} \Omega .
$$

Finally, combining (2.1), 2.2) and 2.3, we end up with

$$
r_{2}^{n-1}-r_{2} \leqslant r_{1}^{n-1}-r_{1}
$$


Since the function $\varphi(r)=r^{n-1}-r$ is strictly increasing on $(1, \infty)$, we conclude that $r_{2} \leqslant r_{1}$. However, from the choice of $r_{1}$ and $r_{2}$ we also have $r_{2} \geqslant r_{1}$. The conclusion is that $r_{1}=r_{2}$, and therefore $\partial \Omega$ has to be a sphere. For $n=2$, carrying out the same arguments with $u_{i}(x)=$ $\frac{-\gamma}{\log \left|r_{i}+(1-R)\right|} \log |x+(1-R)|+\gamma$, we obtain the same conclusion.

REMARK 2.1 Let us point out that the above computation does not work, in principle, as a rigorous proof because there is no way to guarantee, at this moment, that $y_{1}, y_{2} \in \partial_{\text {red }} \Omega$, even though in [23] it is shown that $\mathcal{H}^{n-1}\left(\partial \Omega \backslash \partial_{\text {red }} \Omega\right)=0$. For those who liked this geometric proof, here are good news: there are two ways of making this proof work. The first way is to use the fact that the free boundary condition $u_{v} \equiv \lambda$ also holds in the viscosity sense. In the viscosity theory, the points $y_{1}, y_{2}$ are regular, thus using the linear behavior of $u$ close to the free boundary, we can make inequality (2.2) hold. The second way is to jump ahead in this paper and use Theorem 3.2 to initially ensure full regularity of the free boundary. Then we no longer need to count on luck when using this touching-by-spheres argument.

THEOREM 2.2 (Radial symmetry for the linear problem) Let $D$ be a ball in $\mathbb{R}^{n}$ with radius $R$, and $\Gamma(t)=t$. Then there exists a unique minimizer to Problem 1 and it is radially symmetric. In particular the free boundary is a sphere.

Proof. Our proof uses the Schwarz rearrangement technique (see [18]). Here are some details. Let $A$ be a measurable set. The Schwarz symmetrization of $A$, which we denote by $A^{*}$, is defined by $A^{*}=B(r)$, where $|B(r)|=|A|$. Let $f: \bar{U} \rightarrow \mathbb{R}$ be a function. Set $U_{c}:=\{x \in \bar{U}: u(x) \geqslant c\}$. We define the Schwarz rearrangement of $f$ by $f^{*}(x):=\sup \left\{c \in \mathbb{R}: x \in U_{c}^{*}\right\}$. The crucial information we shall use is that if $f: U \rightarrow \mathbb{R}$ is in $H^{1}(U)$, then

$$
\int_{U}|\nabla f|^{2} \mathrm{~d} x \geqslant \int_{U^{*}}\left|\nabla f^{*}\right|^{2} \mathrm{~d} x
$$

Furthermore, if $\partial U$ is piecewise analytic and $f$ is analytic, then equality holds if and only if $f=$ $f^{*}+c$. For this fact we refer to [15].

Let us return to our original purpose. Let $u$ be a solution to our problem. The first observation is that, in the linear case, i.e., $\Gamma(t)=t$, with constant temperature distribution $\gamma$, if we apply Green's formula, we find

$$
J(u):=\int_{\partial D} u_{\mu} \mathrm{d} \sigma=\gamma^{-1} \int_{\operatorname{supp} u \backslash D}|\nabla u|^{2} \mathrm{~d} x .
$$

Notice that, from [23], $\partial\left[\operatorname{supp} u\right.$ ] is an analytic surface up to an $\mathcal{H}^{n-1}$-negligible set, thus the above computation can be made. Let $u^{*}$ be the Schwarz symmetrization of $u$. Then

- $\left.\left.u^{*}\right|_{\mathbb{S}^{n-1}} \equiv u\right|_{\mathbb{S}^{n-1}} \equiv \gamma$.

- $\operatorname{Vol}\left(\operatorname{supp} u^{*} \backslash B(1)\right)=\operatorname{Vol}(\operatorname{supp} u \backslash B(1))=1$.

Let $h$ denote the harmonic function in supp $u^{*} \backslash B(1)$ with boundary values equal to $\gamma$ on $\mathbb{S}^{n-1}$ and 0 on $\partial\left[\operatorname{supp} u^{*}\right]$. Notice that $h$ competes with $u$ in the minimization problem 1.3 . We then have

$$
\begin{aligned}
J(u) & =\gamma^{-1} \int_{\operatorname{supp} u \backslash B(1)}|\nabla u|^{2} \mathrm{~d} x \geqslant \gamma^{-1} \int_{\operatorname{supp} u^{*} \backslash B(1)}\left|\nabla u^{*}\right|^{2} \mathrm{~d} x \\
& \geqslant \gamma^{-1} \int_{\operatorname{supp} u^{*} \backslash B(1)}|\nabla h|^{2} \mathrm{~d} x=J(h) .
\end{aligned}
$$


However, from the minimization property of $u$, we know $J(u) \leqslant J(h)$. Thus we have equality in the above expression. Therefore, from the previous observation, having in mind that $u=u^{*}$ on $\mathbb{S}^{n-1}$, and $\partial\{u>0\}$ is an analytic surface up to a closed $\mathcal{H}^{n-1}$-negligible set (see [23]), we conclude that $u=u^{*}$, thus $u$ is radial. Since the minimizer $u$ of Problem 1 was taken arbitrarily, we have shown that any solution of Problem 1 with $\Gamma(t)=t$ is radial, in particular, the free boundary is a sphere. From the volume constraint $|\Omega \backslash B(1)|=1$, there must exist a unique solution.

Let us turn our attention to the nonlinear setting, i.e., $\Gamma$ is a generic increasing and convex nonlinearity. It seems acceptable that, even in this situation, the best way of insulating a ball, $B(R)$, is by another ball. Thus, if our conjecture is right, the solution of Problem 1 would not depend upon the nonlinearity $\Gamma$. Set

$$
L(x):= \begin{cases}\frac{\gamma}{R^{2-n}-r_{n}^{2-n}}|x|^{2-n}-\frac{\gamma r_{n}^{2-n}}{R^{2-n}-r_{n}^{2-n}} & \text { if } n \geqslant 3, \\ \frac{-\gamma}{\log \left|r_{2}+(1-R)\right|} \log |x+(1-R)|+\gamma & \text { if } n=2 .\end{cases}
$$

Here

$$
r_{n}:=\sqrt[n]{\frac{\omega_{n}+R^{n}}{\omega_{n}}},
$$

where $\omega_{n}$ stands for the volume of the unit ball in $\mathbb{R}^{n}$, i.e., $\omega_{n}=|B(1)|$. This is the solution of Problem 1 for $\Gamma(t)=t$, provided by Theorem 2.2. Let $u=u_{\Gamma}$ be a solution of Problem 1 for a generic nonlinearity $\Gamma$. Since we want to show $u$ is radially symmetric, it seems natural to try to compare it with $L$ in terms of the optimization problems they minimize. Suppose by contradiction that $u$ is not radially symmetric. In particular $u \neq L$. From Jensen's inequality, we have

$$
\begin{aligned}
J(u) & =\int_{\mathbb{S}^{n-1}} \Gamma\left(u_{\mu}(x)\right) \mathrm{d} \sigma(x) \geqslant n \omega_{n} \Gamma\left(\int_{\mathbb{S}^{n-1}} u_{\mu}(x) \frac{\mathrm{d} \sigma(x)}{n \omega_{n}}\right) \\
& >n \omega_{n} \Gamma\left(\int_{\mathbb{S}^{n-1}} L_{\mu}(x) \frac{\mathrm{d} \sigma(x)}{n \omega_{n}}\right),
\end{aligned}
$$

where in the last inequality we have used the fact that $L$ is the only minimizer to Problem 1 with $\Gamma(t)=t$. However, the geometry of our problem is so special that

$$
L_{\mu}(x) \equiv c_{n}= \begin{cases}\frac{(n-2) \gamma}{R^{2-n}-r_{n}^{2-n}} & \text { if } n \geqslant 3 \\ \frac{\gamma}{\log \left|r_{2}+(1-R)\right|} & \text { if } n=2 .\end{cases}
$$

Returning to inequality $(2.5)$, having in mind the above remark, we conclude

$$
\begin{aligned}
J(u) & >n \omega_{n} \Gamma\left(\int_{\mathbb{S}^{n-1}} L_{\mu}(x) \frac{\mathrm{d} \sigma(x)}{n \omega_{n}}\right)=n \omega_{n} \Gamma\left(c_{n}\right) \\
& =\int_{\mathbb{S}^{n-1}} \Gamma\left(L_{\mu}(x)\right) \mathrm{d} \sigma(x)=J(L) .
\end{aligned}
$$

Since $L$ competes with $u$ in the minimization problem (1.3), the strict inequality in (2.6) leads to a contradiction. Summarizing, we have proven the following general theorem: 
THEOREM 2.3 (Radial symmetry for Problem 1 ) Let $D$ be a ball in $\mathbb{R}^{n}$ and $\Gamma(t)$ be a general convex and increasing nonlinearity. Then there exists a unique minimizer to Problem 1 and it is radially symmetric. In particular, the free boundary is a sphere.

\section{Starlikeness and full regularity of the free boundary}

In the previous section we studied a very special symmetry, namely the spherical symmetry. As we pointed out, this is an extreme symmetry case which enables strong results such as Theorem 2.3 In this section we shall explore a milder restriction on the body $D$. The geometric property we shall be concerned with is starlikeness. Here is its definition.

Definition 3.1 Let $E \subset \mathbb{R}^{n}$ be a set and $x_{0} \in E$. We say $E$ is starlike with respect to $x_{0}$ if for any $x \in E$ and any $t \in[0,1]$, we have $t x_{0}+(1-t) x \in E$.

For instance, any convex body is starlike with respect to all of its points. Even assuming a less restrictive assumption, we shall be able, in the linear setting, to prove uniqueness and full regularity of the free boundary. The main theorem of this section is the following.

THEOREM 3.2 Let $D \subset \mathbb{R}^{n}$ be starlike with respect to any point of a small neighborhood of the origin, say $B_{\varepsilon}(0)$, and let $\Gamma(t)=t$. Then there exists a unique minimizer $u$ of Problem 1 . Moreover the level sets $\{u>\alpha\}$ are starlike with respect to $B_{\varepsilon}(0)$. Furthermore the free boundary is an analytic surface with no singular points, i.e., $\partial_{\text {red }}\{u>0\}=\partial\{u>0\}$.

The proof of this theorem will be developed throughout this section. The idea is to consider a "starlike version" of $u$ given by an appropriate rearrangement and compare it with $u$ and with solutions to auxiliary free boundary problems.

It is worth pointing out that the linear setting of our problem is closely related to Bernoulli-type problems that have been studied by many authors. For the Bernoulli problem on convex domains, a Perron-type method has been successfully applied to prove existence, uniqueness and convexity of the solution [13, 14]. Acker has proven some deep results about solutions of the Bernoulli problem under convexity and starlikeness assumptions (see, for instance, [1, 2]). One of the major difficulties to properly relate Problem 1 to a Bernoulli-type problem, though, is that, for $n \geqslant 3$, one cannot ensure full regularity of the free boundary of our original variational problem. Our strategy will then be to compare a special solution of Problem 1 , whose free boundary can be shown to be fully regular, to solutions of certain Bernoulli problems. Once full regularity of the free boundary of a solution of Problem 1 is established, we will be able to apply the usual techniques employed in Bernoulli-type problems. Indeed, Lemma 3.6 is essentially due to Acker and Meyer in [3]. Lemma 3.7 is inspired by [15], while Theorem 3.11 is an adaptation of a beautiful geometric argument due to Henrot and Shahgholian in [12]. For completeness, we shall carry out all the details and necessary modifications.

Hereafter, we fix a solution $u$ to Problem 1 with $\Gamma(t)=t$ and we assume that $D \subset \mathbb{R}^{n}$ is starlike with respect to any point in $B_{\varepsilon}(0)$. As pointed out before, there exists a constant $\lambda=\lambda(u)$ such that the following free boundary condition holds:

$$
u_{v} \equiv \lambda \quad \text { on } \partial_{\text {red }}\{u>0\} .
$$

This leads us to consider the following two auxiliary free boundary problems: 
Problem 2 (Alt-Caffarelli) Minimize

$$
J(v):=\int_{D^{C}}\left(|\nabla v|^{2}+\lambda^{2} \chi_{\{v>0\}}\right) \mathrm{d} x
$$

over $\left\{v \in H^{1}\left(D^{C}\right): v \equiv \gamma\right.$ on $\left.\partial D\right\}$.

PRoblem 3 (Bernoulli free boundary problem) Find a domain $\Omega \supset D$ and a function $\psi$ such that

$$
\begin{cases}\Delta \psi=0 & \text { in } \Omega \backslash D \\ \psi=\gamma & \text { on } \partial D \\ \psi=0, & |\nabla \psi|=\lambda\end{cases}
$$

For completeness let us briefly discuss the starlike rearrangement we shall make use of. A point $x \in \mathbb{R}^{n}$ can be represented in spherical coordinates as $\left(r, \theta_{1}, \ldots, \theta_{n-1}\right)$, where $r \in \mathbb{R}_{0}^{+}$and $\theta=$ $\left(\theta_{1}, \ldots, \theta_{n-1}\right) \in T:=[0, \pi]^{n-2} \times[-\pi, \pi]$. Let $E$ be a bounded set containing $B_{\varepsilon}(0)$ and define $g_{p}(r)=r^{n-1-p}$. Let $G_{p}(r)=\frac{1}{n-p} r^{n-p}$ be a primitive of $g_{p}$. We define

$$
\begin{aligned}
E(\theta) & :=\{r \geqslant \varepsilon:(r, \theta) \in E\}, \\
l(\theta) & :=\int_{E(\theta)} g_{p}(r) \mathrm{d} r, \\
h(\theta) & :=l(\theta)+G_{p}(\varepsilon), \\
R(\theta) & :=G_{p}^{-1}(h(\theta)) .
\end{aligned}
$$

We then define the p-starlike rearrangement of $E$ by

$$
E_{p}^{*}:=\left\{(r, \theta) \in \mathbb{R}_{0}^{+} \times T: 0 \leqslant r \leqslant R(\theta)\right\}
$$

Notice that, in our case, if we extend $u$ by $\gamma$ inside of $D$, from the maximum principle, $u$ attains its maximum, namely $\gamma$, at each point of $B_{\varepsilon}(0)$. Thus, any level set $\{u>\alpha\}$ contains $B_{\varepsilon}(0)$ and so it is suitable for starlike rearrangement.

We finally define the $p$-decreasing starlike rearrangement of $u$, denoted $u_{p}^{*}$, by

$$
u_{p}^{*}(x):= \begin{cases}\sup \left\{\alpha \in \mathbb{R}_{0}^{+}: x \in\{u>\alpha\}_{p}^{*}\right\} & \text { for } x \in[\operatorname{supp} u]_{p}^{*} \\ 0 & \text { otherwise. }\end{cases}
$$

We define $u^{*}=u_{2}^{*}$. We shall use the following powerful result.

THEOREM 3.3 (Property of the starlike rearrangement) In our setting,

(1) $\operatorname{Vol}\left(\left\{u^{*}>0\right\}\right) \leqslant 1$.

(2) $\int_{D^{C}}\left|\nabla u^{*}\right|^{2} \mathrm{~d} x \leqslant \int_{D^{C}}|\nabla u|^{2} \mathrm{~d} x$.

The first part of this theorem follows from the fact that for any domain $E \supset B_{\varepsilon}(0), \operatorname{Vol}(E)=$ $\operatorname{Vol}\left(E_{0}^{*}\right)$ together with the inequality $u_{0}^{*} \geqslant u_{p}^{*}$ for any $p \geqslant 1$. The second part follows from the general fact that if $v \in W_{0}^{1, p}(U)$ then $\int_{U}|\nabla v|^{p} \mathrm{~d} x \geqslant \int_{U_{p}^{*}}\left|\nabla v_{p}^{*}\right|^{p} \mathrm{~d} x$. For the proofs of these facts and further details, we refer to [15]. We need some lemmas. 
LEMMA 3.4 Under the hypothesis of Theorem 3.2 and the notation above, $u^{*}$ is a minimizer of Problem 1. Furthermore the free boundary $\partial\left\{u^{*}>0\right\}$ is an analytic surface with no singular points, i.e., $\partial_{\text {red }}\left\{u^{*}>0\right\}=\partial\left\{u^{*}>0\right\}$.

Proof. Notice that, due to the volume constraint, in principle $u^{*}$ does not compete with $u$ in Problem 1. To overcome this difficulty, we shall use Theorem 1.2 . Consider the functional

$$
J_{\varepsilon}(\xi):=\int_{\partial D} \xi_{\mu}(x) \mathrm{d} \sigma+f_{\varepsilon}(|\{\xi>0\}|),
$$

for $\varepsilon$ small enough so that Theorem 1.2 applies. Let $h$ be the harmonic function equal to $\gamma$ on $\partial D$ and 0 on $\partial\left\{u^{*}>0\right\}$. From Theorem 3.3 , we obtain

$$
\begin{aligned}
J_{\varepsilon}(u) & =\gamma^{-1} \int_{D^{C}}|\nabla u(x)|^{2} \mathrm{~d} x+1 \geqslant \gamma^{-1} \int_{D^{C}}\left|\nabla u^{*}(x)\right|^{2} \mathrm{~d} x+1 \\
& \geqslant \gamma^{-1} \int_{D^{C}}|\nabla h(x)|^{2} \mathrm{~d} x+1 \geqslant J_{\varepsilon}(h) \geqslant J_{\varepsilon}(u) .
\end{aligned}
$$

We conclude that, first of all, $u^{*}=h$. Furthermore, it is a minimizer to the penalized version of Problem 11 Hence, from Theorem 1.2, $u^{*}$ is a solution to Problem 1. In particular, we have $\operatorname{Vol}\left(\left\{u^{*}>0\right\} \backslash D\right)=1$.

For the second part of the lemma, we notice that, since $\left\{u^{*}>0\right\}$ is starlike with respect to all points in $B_{\varepsilon}(0)$, for each $z \in \partial\left\{u^{*}>0\right\}$ there exists a cone $C$ that contains $B_{\varepsilon}(0)$ such that $\partial\left\{u^{*}>0\right\} \cap C=\{x\}$. This proves that the free boundary $\partial\left\{u^{*}>0\right\}$ is Lipschitz. Moreover, since $u^{*}$ is a minimizer to Problem $1, \Delta u^{*}=0$ in $\left\{x \in D^{C}: u^{*}>0\right\}$ and there exists a constant $\lambda^{*}>0$ such that

$$
u_{v}^{*} \equiv-\lambda^{*} \quad \text { on } \partial_{\text {red }}\left\{u^{*}>0\right\}
$$

Now, as we have already anticipated, we can interpret our free boundary condition in the viscosity sense (for a more general situation, where degenerate operators govern the system, see [19]). Thus, we can apply Caffarelli's theorem in [8] (Lipschitz free boundaries are $C^{1, \alpha}$ ) to conclude that $\partial\left\{u^{*}>0\right\}$ is a $C^{1, \alpha}$ surface. Afterwards, having in mind our free boundary condition, by [16] we deduce the analyticity of the free boundary $\partial\left\{u^{*}>0\right\}$.

We now turn our attention to Problem 3 , with $\lambda$ replaced by $\lambda^{*}$. We need some notations. For any set $A \subset \mathbb{R}^{n}$, and $t>0$, we set $t A=\{t x: x \in A\}$. Let $\mathbb{V}$ be the set of all bounded simply connected domains $U \subset \mathbb{R}^{n}$ containing the origin, and denote by $\partial \mathbb{V}$ the boundaries of elements in $\mathbb{V}$. In $\partial \mathbb{V}$ we define the metric

$$
\rho\left(S_{1}, S_{2}\right):=\sup \left\{|\log t|: t S_{1} \cap S_{2} \neq \emptyset\right\} .
$$

For each $S \in \partial \mathbb{V}$ let $U \in \mathbb{V}$ be the bounded simply connected domain for which $\partial U=S$. We say $S_{1} \leqslant S_{2}$ if $U_{1} \subset U_{2}$ and $S_{1}<S_{2}$ if $\bar{U}_{1} \subset U_{2}$, where $U_{i} \in \mathbb{V}$ and $\partial U_{i}=S_{i}$ for $i=1$, 2. In what follows, we will need the Lavrent'ev principle. A proof can be found in [17].

LEMMA 3.5 (Lavrent'ev principle) Let $S_{1}, S_{2}$ and $\tilde{S}_{1}, \tilde{S}_{2}$ be $n-1$-hypersurfaces in $\partial \mathbb{V}$. Let $\Omega$ (resp. $\tilde{\Omega}$ ) be the annular domain whose boundaries are $S_{1}$ and $S_{2}$ (resp. $\tilde{S}_{1}$ and $\tilde{S}_{2}$ ) and let $U$ (resp. $\tilde{U})$ be the harmonic function in $\Omega(\operatorname{resp} . \tilde{\Omega})$, equal to 1 on $S_{1}\left(\operatorname{resp} . \tilde{S}_{1}\right)$ and 0 on $S_{2}\left(\operatorname{resp} . \tilde{S}_{2}\right)$. Let $t \geqslant 1$ be such that $S_{1} \leqslant t \tilde{S}_{1}$ and $S_{2} \leqslant t \tilde{S}_{2}$. If $t x_{i} \in S_{i} \cap t \tilde{S}_{i}$ and $\left|\nabla U_{i}\left(x_{i}\right)\right|$ exist, then

$$
\left|\nabla \tilde{U}\left(x_{2}\right)\right| \geqslant t\left|\nabla U\left(t x_{2}\right)\right| \text { and }\left|\nabla \tilde{U}\left(x_{1}\right)\right| \leqslant t\left|\nabla U\left(t x_{1}\right)\right| .
$$


The next lemma concerns uniqueness. We observe that the main difficulty of uniqueness results for free boundary variational problems is the lack of regularity of the free boundary, as pointed out in the "fake" proof of radial symmetry presented in Section 2. The advantage of dealing with Problem 3 is that solutions are supposed to be full regular. The next lemma is a consequence of the Lavrent'ev principle.

Lemma 3.6 The function $u^{*}$ is the only classical solution to Problem 3 where $\lambda$ is replaced by $\lambda^{*}$.

Proof. From Lemma 3.4, $u^{*}$ is a classical solution to Problem 3 , where $\lambda$ is replaced by $\lambda^{*}$. Let $(\psi, \Omega)$ be another solution to Problem 3 Define $S=\partial \Omega$ and $F\left(u^{*}\right)=\partial\left\{u^{*}>0\right\}$. If $(\psi, \Omega) \neq$ $\left(u^{*},\left\{u^{*}>0\right\}\right)$, there would exist a $t_{0}>1$ such that $\log t_{0}=\rho\left(S, F\left(u^{*}\right)\right)$. Thus, $S \leqslant t_{0} F\left(u^{*}\right)$ and $F\left(u^{*}\right) \leqslant t_{0} S$. Furthermore, either $S \cap t_{0} F\left(u^{*}\right) \neq \emptyset$ or $t_{0} S \cap F\left(u^{*}\right) \neq \emptyset$. We might assume without loss of generality that there exists a point $t_{0} x_{0} \in S \cap t_{0} F\left(u^{*}\right)$ (if $t_{0} S \cap F\left(u^{*}\right) \neq \emptyset$ we proceed similarly). Notice that, since our fixed domain $D$ is assumed to be starlike, we have $\partial D \leqslant t_{0} \partial D$. Finally, applying Lemma 3.5 for $S_{1}=\tilde{S}_{1}=\partial D, S_{2}=S, \tilde{S}_{2}=F\left(u^{*}\right)$ and $t=t_{0}$, we obtain

$$
\lambda^{*}=\left|\nabla u^{*}\left(x_{0}\right)\right| \geqslant t_{0}\left|\nabla \psi\left(t_{0} x_{0}\right)\right|=t_{0} \lambda^{*},
$$

which is a contradiction. Thus, $S$ has to be $F\left(u^{*}\right)$ and therefore the lemma is proven.

Our next step is to relate our original problem to Problem 2 and the latter to Problem 3 . We stress that the advantage of dealing with Problem 2 instead of our original Problem 1 is that the former does not have volume constraint. This makes it much easier to construct perturbation arguments as will be explicit in the proof of the next lemma.

Lemma 3.7 Let $v$ be a minimizer of Problem 2 with $\lambda$ replaced by $\lambda^{*}$. Then the free boundary $\partial\{v>0\}$ is an analytic surface with no singular points.

Proof. We follow the same idea of the proof of Lemma 3.4. Let $v$ be a minimizer of Problem 2 with $\lambda$ replaced by $\lambda^{*}$. Let $v^{*}$ be the 2-decreasing starlike rearrangement of $v$. As in Lemma 3.4 we easily conclude, with the aid of Theorem 3.3 , that $v^{*}$ is also a minimizer of Problem 2. Define $v^{+}:=\max \left\{v, v^{*}\right\}$ and $v^{-}:=\min \left\{v, v^{*}\right\}$. Notice that $v^{+}$and $v^{-}$are admissible for Problem 2 (not for Problem 1 or even for its penalized version, though). Furthermore, we easily observe that

$$
J\left(v^{+}\right)+J\left(v^{-}\right)=J(v)+J\left(v^{*}\right)=2 \min J .
$$

Thus $v^{+}$and $v^{-}$are also minimizers of Problem 2 Let $\Omega^{-}:=\left\{v^{-}>0\right\} \backslash D$. Notice that

$$
\Delta\left(v^{+}-v\right)=\Delta\left(v^{+}-v^{*}\right)=0 \quad \text { in } \Omega^{-} .
$$

Moreover, $v^{+}-v \geqslant 0$ in $\partial \Omega^{-}$and $v^{+}-v^{*} \geqslant 0$ in $\Omega^{-}$. From the maximum principle, we conclude that either

(1) $v^{*}=v$ in $\Omega^{-}$, or

(2) $v^{*}>v$ in $\Omega^{-}$, or

(3) $v>v^{*}$ in $\Omega^{-}$.

Notice that, if (2) happens, then, by the continuity of $v^{*}$ it must be the case that $\operatorname{supp} v \subsetneq \operatorname{supp} v^{*}$. But this would violate Theorem 3.3. If (3) happens, then, again by the continuity of $v, \operatorname{supp} v^{*} \subsetneq$ $\operatorname{supp} v$. However, if this is the case, we would conclude that $J\left(v^{*}\right)<J(v)$, a contradiction. Thus, 
we conclude that $v^{*}=v$ in $\Omega^{-}$, which clearly implies $v^{*}=v$ and therefore $v$ is starlike with respect to $B_{\varepsilon}(0)$.

Now, by the same argument as in Lemma 3.4, we obtain the full regularity of the free boundary $\partial\{v>0\}$. Indeed, the fact that the free boundary is starlike with respect to $B_{\varepsilon}(0)$ implies its Lipschitz regularity. Furthermore, from [9], $v$ satisfies the free boundary condition

$$
v_{v} \equiv-\lambda^{*} \quad \text { on } \partial_{\text {red }}\{v>0\}
$$

in the viscosity sense. Invoking [8] and afterwards [16], we deduce the analyticity of the whole free boundary. Since $v$ was taken to be an arbitrary minimizer of Problem 2, the proof is finished.

The next lemma unifies the results we have so far by relating Problem 1 and Problem 2 . As we shall see, from the full regularity results we have obtained, we shall be able to relate these problems to Problem 3 where we could obtain a uniqueness result. As already pointed out a couple of times, uniqueness of variational problems with free boundary is, in general, quite hard, so the idea is to use the auxiliary Problem 3, where, from Lemma 3.6, we have uniqueness of classical solutions, and compare $u^{*}$ with a solution of Problem 2 , in terms of Problem 3 .

LEMma 3.8 With the hypothesis of Theorem 3.2 and the notation previously set, the function $u^{*}$ is the only minimizer to Problem 2 with $\lambda$ replaced by $\lambda^{*}$.

Proof. From Lemma 3.4 $\partial\left\{u^{*}>0\right\}$ is a smooth surface and from the free boundary condition, we have $u_{v}^{*} \equiv-\lambda^{*}$ on $\partial\left\{u^{*}>0\right\}$. Lemma 3.7 guarantees the full regularity of the free boundary $\partial\{v>0\}$ of any solution $v$ of Problem 2 with $\lambda$ replaced by $\lambda^{*}$. Furthermore, from the free boundary condition established in [7], we also have $v_{v} \equiv-\lambda^{*}$ on $\partial\{v>0\}$. We conclude that both $u^{*}$ and $v$ are classical solutions to Problem 3 . Lemma 3.6 finally implies $u^{*}=v$.

We are ready to make the last step toward the proof of Theorem 3.2 Basically, the only information left is whether $u$ is equal to its rearrangement.

Lemma 3.9 With the hypothesis of Theorem 3.2 and the notation previously set, the function $u^{*}$ is the only solution to Problem 1

Proof. Again, it seems quite hard to show this directly. We will use Problem 2 to compare a solution $\omega$ of Problem 1 and $u^{*}$. Indeed, consider the functional in Problem 2 with $\lambda$ replaced by $\lambda^{*}$, i.e.,

$$
J(v):=\int_{D^{C}}\left(|\nabla v|^{2}+\left(\lambda^{*}\right)^{2} \chi_{\{v>0\}}\right) \mathrm{d} x .
$$

We have shown that $u^{*}$ is the unique minimizer of $J$ in $\left\{v \in H^{1}\left(D^{C}\right): v \equiv \gamma\right.$ on $\left.\partial D\right\}$. Furthermore, we have verified that $\operatorname{Vol}\left(\left\{u^{*}>0\right\} \backslash D\right)=1$. Thus, if $\omega$ is any solution to Problem 1 , then

$$
J(\omega)=\int_{D^{C}}|\nabla \omega|^{2} \mathrm{~d} x+\left(\lambda^{*}\right)^{2}=\int_{D^{C}}\left|\nabla u^{*}\right|^{2} \mathrm{~d} x+\left(\lambda^{*}\right)^{2}=J\left(u^{*}\right) .
$$

Lemma 3.8 implies $\omega=u^{*}$. Since $\omega$ was taken to be an arbitrary solution to Problem 1 . Lemma 3.9 is proven.

Finally, combining Lemmas 3.4 and 3.9 we conclude the proof of Theorem 3.2 . 
REMARK 3.10 As pointed out in the introduction, full regularity of the free boundary of minimizers of Problem 2, without any further assumption on $\partial D$, is a very challenging question. It has been proven that in dimensions 2 and 3 the singular set on the free boundary of minimizers of Problem 2 is empty. Furthermore, there are indications that this should be the case for any dimension up to 6; however, there is a prediction that one could find a counterexample in dimension 7 . The full regularity of the free boundary in Theorem 3.2, and its impact in Lemma 3.7, are possible because we impose a geometric restriction on $\partial D$.

Let us mention that, from the machinery developed above, one can infer the convexity of the free boundary in the case where $D$ is a convex body.

THEOREM 3.11 Under the hypothesis of Theorem 3.2, if $D$ is a convex body, then so is any level set $\{u>\alpha\}$ of the minimizer of Problem 1 .

Proof. Since $D$ is convex, it is starlike with respect to all of its points. From Theorem 3.2, there exists a unique solution to Problem 11, its level sets are starlike with respect to all points in $D$, and the free boundary $\partial\{u>0\}$ is a smooth surface with no singular points. In particular, the free boundary condition $-u_{v} \equiv \lambda>0$ holds at any point of the free boundary.

Set $\Omega=\operatorname{supp} u$, let $\Omega^{\star}:=\overline{c o}(\Omega)$ be the convex hull of $\Omega$, and $u^{\star}$ the harmonic function on $\Omega^{\star} \backslash D$, equal to $\gamma$ on $\partial D$ and 0 on $\partial \Omega^{\star}$. Define $U_{c}=\left\{x \in \Omega^{\star}: u^{\star}(x) \geqslant c\right\}$. From [11], these are convex sets. Let $y$ be a generic point on $\partial U_{c}$. We may assume, with no loss of generality, that $y$ is the origin and the exterior normal derivative at 0 is $e_{1}$. Let $z \in \partial \Omega^{\star} \cap\left\{x_{1}>0\right\}$ be the farthest point to $\left\{x_{1}=0\right\}$. Notice that, since $\Omega^{\star}=\overline{\operatorname{co}}(\Omega), z$ can be taken to belong to $\partial \Omega \cap \partial \Omega^{\star}$. On the open set $\Omega^{\star} \cap\left\{x_{1}>0\right\}$, consider the harmonic function $v(x)=u^{\star}(x)+\beta\left\langle x, e_{1}\right\rangle$ with $0<\beta<\lambda$. By the maximum principle, $v$ achieves its maximum either at $z$ or at 0 .

We claim that $v$ attains its maximum at the origin. Indeed, let $B=B\left(z+e_{1}, 1\right)$. By Lemma 3.8 of the present paper, Lemma 4.10 in [7] and the maximum principle, there exists a sequence $z_{n} \rightarrow z$ such that $\lim _{n \rightarrow \infty} u^{\star}\left(z_{n}\right) / \operatorname{dist}\left(z_{n}, B\right) \geqslant \lambda$. If $v$ achieved its maximum at $z$, we would have

$$
0 \geqslant \frac{v\left(z_{n}\right)-v(z)}{\operatorname{dist}\left(z_{n}, B\right)} \geqslant \frac{u^{\star}\left(z_{n}\right)}{\operatorname{dist}\left(z_{n}, B\right)}+\beta \frac{\left\langle z_{n}-z, e_{1}\right\rangle}{\operatorname{dist}\left(z_{n}, B\right)} \geqslant \lambda+O(n)-\beta,
$$

which contradicts the choice of $\beta$.

Thus, $v$ attains its maximum at the origin. But this implies $\left|\nabla u^{\star}(0)\right| \geqslant \beta$. Since $\beta<\lambda$ and $y$ were taken arbitrarily, we conclude that $\left|\nabla u^{\star}(x)\right| \geqslant \lambda$ for all $x \in \Omega^{\star} \backslash D$.

Now, the assertion of Theorem 3.11 follows as a consequence of Lemma 3.5. Indeed, with the notation previously set, for $0<t<1$, consider $t \Omega^{\star}=\left\{t x: x \in \Omega^{\star}\right\}$, let $t_{0}:=\sup \left\{t: t \Omega^{\star} \subset \Omega\right\}$ and $\chi \in \partial t \Omega^{\star} \cap \partial \Omega$. Applying Lemma 3.5. we obtain

$$
\lambda=|\nabla u(\chi)| \geqslant \frac{1}{t_{0}}\left|\nabla u^{\star}\left(\chi / t_{0}\right)\right| \geqslant \frac{1}{t_{0}} \lambda .
$$

In conclusion, $\Omega^{\star}=\Omega$. Hence, the optimal configuration supp $u$ is convex and then, from [11], so is any level set $\{u>\alpha\}$.

\section{Uniqueness and $O(n)$ symmetries}

Let $u$ and $v$ be two different solutions of our general free boundary variational problem (1.1). Since $u \equiv v$ on $\partial D$ and both are harmonic near it, if $u_{\mu}$ coincides with $v_{\mu}$ on any tiny open subset of $\partial D$, 
then $u \equiv v$ on the whole $D^{C}$. On the other hand, the set $\Theta:=\left\{\chi \in \partial D \mid u_{\mu}(\chi)=v_{\mu}(\chi)\right\}$ has to be nonempty, otherwise, say $u_{\mu}>v_{\mu}$ in $\partial D$ and, since $\Gamma$ is increasing, $\int_{\partial D} \Gamma\left(x, u_{\mu}(x)\right) \mathrm{d} S(x)>$ $\int_{\partial D} \Gamma\left(x, v_{\mu}(x)\right) \mathrm{d} S(x)$. Moreover, for any domain $U$ surrounding $D$, i.e., $D \subset U$, there must exist a $\xi \in \partial U$ such that $u(\xi)=v(\xi)$; otherwise, by the maximum principle, say $v>u$ in $U \backslash D$ and therefore, $v_{\mu}<u_{\mu}$ on some $\mathcal{H}^{n-1}$-noneligible subset of $\partial D$, and thus $\int_{\partial D} \Gamma\left(x, u_{\mu}(x)\right) \mathrm{d} S(x)>$ $\int_{\partial D} \Gamma\left(x, v_{\mu}(x)\right) \mathrm{d} S(x)$. In $\mathbb{R}^{2}$, the above comment provides a nice stratification of the set $\{u>0\}$ $\cap\{v>0\}$ dividing it into regions where $u>v$ and $v>u$. In other words, there are curves $\gamma_{i}$ joining points of $\partial\{u>0\} \cap \partial\{v>0\}$ and $\partial D$, so that $u \equiv v$ along $\gamma_{i}$.

The above paragraph brings some general information that, depending upon the geometry of $D$, might yield uniqueness results. We should point out, though, that to establish a general uniqueness result, if true, seems to be a very hard task. Symmetry results for the general nonlinear variational free boundary problem 1.1, or even its simplified version, Problem 1], seem also very challenging. For instance, one of the most common ways of proving symmetry results is by rearrangement techniques. That was our strategy to deliver the results in the previous section as well as to obtain the starting radial symmetry result for the linear setting in Section 2. Notice that rearrangement techniques work fine in the linear setting of our problem, i.e., when $\Gamma(t)=t$ with constant temperature distribution, $\phi \equiv \gamma$. That is because, in this situation, our functional is equivalent to the energy functional and if one minimizes the latter, it will automatically be harmonic in its set of positivity. For the general problem, one has to search for minimizers among subharmonic functions; see for instance, the penalized version (1.2). Now, if $u$ is an admissible function for Problem (1.2), it seems hard to control the sign of the Laplacian of its rearrangement. Another difficulty in using rearrangement techniques for the nonlinear problem is that our functional is equivalent to

$$
\mathfrak{J}(u):=\int_{D^{C}}\left(2 u \Upsilon^{\prime}\left(|\nabla u|^{2}\right)\left\langle D^{2} u \cdot \nabla u, \nabla u\right\rangle+\Gamma(|\nabla u|)|\nabla u|+u \Upsilon\left(|\nabla u|^{2}\right) \Delta u\right) \mathrm{d} x,
$$

where $\Upsilon\left(t^{2}\right) t=\Gamma(t)$. The functional $\mathfrak{J}$ does not seem to behave well under rearrangements. Thus, in order to establish symmetry results for Problem [1.1), we have to search for a different type of geometric argument other than rearrangements.

The nonlinearity $\Gamma$ reflects in a rather nonlocal free boundary condition given by (see [23])

$$
\int_{\partial D} \partial_{t} \Gamma\left(x, u_{\mu}(x)\right)\left[H_{\nu}(x, y) u_{v}(y)\right] \equiv \text { const },
$$

for any $y \in \partial_{\text {red }}\{u>0\}$. Here $H$ is a kernel related to the Green's function of the domain $\{u>0\} \backslash D$. Of course, in general, for different nonlinearities, say $\Gamma_{1}$ and $\Gamma_{2}$, there should exist different optimal configurations $u_{1}$ and $u_{2}$. In other words, what happens in the radial symmetry case is very special. Indeed, we can state the following proposition.

Proposition 4.1 Suppose Problem 1.1 admits an optimal configuration $u^{\ell}$, for $\Gamma(t)=t$, that satisfies

$$
u_{\mu}^{\ell} \equiv c \quad \text { on } \partial D .
$$

Assume $\mathcal{H}^{n-1}(\partial D)=1$. Then $u^{\ell}$ is the only solution of Problem 1.1 for any strictly convex nonlinearity $\Gamma(t)$.

Proof. Let $u^{\Gamma}$ be a solution of Problem 1.1. Our first claim is that, necessarily,

$$
u_{\mu}^{\Gamma} \equiv c_{\Gamma} \quad \text { on } \partial D .
$$


Indeed, suppose not. From Jensen's inequality, the minimality properties of $u^{\Gamma}$ and $u^{\ell}$, the strict convexity of $\Gamma$ and 4.2 , we obtain

$$
\begin{aligned}
\int_{\partial D} \Gamma\left(u_{\mu}^{\ell}\right) \mathrm{d} \mathcal{H}^{n-1} & =\Gamma\left(\int_{\partial D} u_{\mu}^{\ell} \mathrm{d} \mathcal{H}^{n-1}\right) \leqslant \Gamma\left(\int_{\partial D} u_{\mu}^{\Gamma} \mathrm{d} \mathcal{H}^{n-1}\right) \\
& <\int_{\partial D} \Gamma\left(u_{\mu}^{\Gamma}\right) \mathrm{d} \mathcal{H}^{n-1} \leqslant \int_{\partial D} \Gamma\left(u_{\mu}^{\ell}\right) \mathrm{d} \mathcal{H}^{n-1}
\end{aligned}
$$

which is a contradiction. This also shows that $u^{\ell}$ is a solution of Problem $\sqrt[1.1]{1}$ for any strictly convex nonlinearity $\Gamma$. If so, taking into account that $u_{\mu}^{\Gamma} \equiv c_{\Gamma}$, we obtain

$$
\Gamma(c)=\Gamma\left(c_{\Gamma}\right),
$$

which implies, as $\Gamma$ is strictly increasing, $c=c_{\Gamma}$, and therefore from the observation made at the beginning of this section, $u^{\ell} \equiv u^{\Gamma}$ in $D^{C}$.

It is worth pointing out that for Problem 1, i.e., for a constant temperature distribution $\phi \equiv \gamma$, a Serrin-type result ensures that the above phenomenon occurs only when $D$ is a ball. For fully regular free boundaries, a proof of this fact can be found in [20] (see also [21]). A stability result pertaining to this remark can be found in [4]. It is also interesting to notice that, when $D$ is convex, we can always, by a scaling argument, assume $\mathcal{H}^{n-1}(\partial D)=1$.

The type of symmetry we will be particularly interested in for the rest of this section is invariance under the group $O(n)$. Let $\mathcal{O}$ be an orthogonal transformation in $O(n)$. Clearly, if we hope to have any kind of symmetry with respect to $\mathcal{O}$ of the free boundary, it is better to assume $\mathcal{O}(D)=D$, $\phi(\mathcal{O}(x))=\phi(x)$ and $\Gamma(\mathcal{O}(x), t)=\Gamma(x, t)$. Under these natural assumptions, we obtain

TheOREM $4.2(O(n)$ symmetries) Let $\mathcal{O} \in O(n)$ be an orthogonal transformation. Suppose Problem 1.1 has a unique solution. Then $u$ is invariant under $\mathcal{O}$.

Proof. Let $u$ be the minimizer of Problem (1.1). Define

$$
u_{\mathcal{O}}(x):=u(\mathcal{O} x)
$$

Since $\mathcal{O}$ preserves volume, one concludes easily that

$$
\left|\operatorname{supp} u_{\mathcal{O}}\right|=|\operatorname{supp} u|=1 \text {. }
$$

We also verify, since $\mathcal{O}(D)=D$ and $\phi(\mathcal{O}(x))=\phi(x)$, that $u_{\mathcal{O}}(x)=\phi(x)$ on $\partial D$. Furthermore

$$
\Delta u_{\mathcal{O}}(x)=0 \quad \text { in } \operatorname{supp} u_{\mathcal{O}}, \quad \nabla u_{\mathcal{O}}(x)=\mathcal{O}^{\mathrm{T}} \nabla u(\mathcal{O} x) .
$$

Thus, $u_{\mathcal{O}}$ competes with $u$ in Problem $[1.1$. From the fact that $|\operatorname{det}(\mathcal{O})|=1$, we obtain, by the change of variables theorem,

$$
\begin{aligned}
J\left(u_{\mathcal{O}}\right) & =\int_{\partial D} \Gamma\left(\left\langle\nabla u_{\mathcal{O}}(x), \mu(x)\right\rangle\right) \mathrm{d} \sigma=\int_{\partial D} \Gamma(\langle\nabla u(\mathcal{O} x), \mathcal{O} \mu(x)\rangle) \mathrm{d} \sigma \\
& =\int_{\partial D} \Gamma(\langle\nabla u(y), \mu(y)\rangle) \mathrm{d} \sigma=J(u)
\end{aligned}
$$

From the uniqueness assumed, $u_{\mathcal{O}} \equiv u$. Therefore, any level set of $u$ is invariant under $\mathcal{O}$ and in particular so is the free boundary. 
COROLlary 4.3 Assume $D$ is starlike with respect to a small neighborhood of the origin and $\Gamma(t)=t$. Suppose $D$ is symmetric with respect to the plane $P=\left\{x_{1}=0\right\}$. Then the solution to Problem 1 is symmetric with respect to the plane $P$ and so is its free boundary.

Proof. Consider the orthogonal transformation

$$
\mathcal{O}\left(x_{1}, x_{2}, \ldots, x_{n}\right):=\left(-x_{1}, x_{2}, \ldots, x_{n}\right) .
$$

Apply Theorem 3.2 and afterwards Theorem 4.2

We finish this paper with some remarks. It seems to us that the nonuniqueness phenomenon for Problem 1 is more related to the topology/geometry of the fixed domain $D$ than to the nonlinearity $\Gamma$. We are therefore led to state the following conjecture:

Conjecture 1 Let $D \subset \mathbb{R}^{n}$ be a domain. Assume there exists a unique solution $u_{\ell}$ to Problem 1 with $\Gamma(t)=t$. Then, for any other strictly convex increasing nonlinearity $\Gamma$, Problem 1 has a unique solution $u_{\Gamma}$.

Another natural conjecture is convexity of the free boundary for Problem 11, with a general nonlinearity $\Gamma$.

CONJECTURe 2 Let $D \subset \mathbb{R}^{n}$ be a convex domain. Then there exists a convex optimal configuration $\{u>0\}$ of Problem 1

Notice that, if Conjecture 2 is affirmatively answered, we would obtain Lipschitz regularity of the full free boundary. From [23], the reduced free boundary is an analytic surface; however, the nonlocal free boundary condition (4.1) does not allow us to deduce, via Caffarelli's viscosity theory, full $C^{1, \alpha}$ regularity of the free boundary, even though it seems quite reasonable to expect it.

Question 1 Let $D \subset \mathbb{R}^{n}$ be a convex domain. Assume Problem 1 has a unique solution. Is the free boundary fully regular?

\section{Acknowledgments}

The author would like to thank Prof. Luis A. Caffarelli for his encouragement and support. He also thanks Luis Silvestre for some helpful discussions. This work was partially supported by CNPqBrazil.

\section{REFERENCES}

1. ACKer, A. A free boundary optimization problem. SIAM J. Math. Anal. 9 (1978), 1179-1191. Zbl 0335.31002 MR 0512520

2. ACKer, A. A free boundary optimization problem. II. SIAM J. Math. Anal. 11 (1980), 201-209. Zbl 0442.31002 MR 0556510

3. ACKER, A., \& MEYER, R. A free boundary problem for the $p$-Laplacian: uniqueness, convexity, and successive approximation of solutions. Electron. J. Differential Equations 1995, no. 8, approx. 20 pp. Zbl 0820.35037 MR 1334863

4. Aftalion, A., BusCa, J, \& Reichel, W. Approximate radial symmetry for overdetermined boundary value problems. Adv. Differential Equations 4 (1999), 907-932. Zbl 0951.35046 MR 1729395

5. Aguilera, N., Alt, H., \& CAfFArelli, L. An optimization problem with volume constraint. SIAM J. Control Optim. 24 (1986), 191-198. Zbl 0588.49005 MR 0826512 
6. Aguilera, N. E., Caffarelli, L. A., \& SprucK, J. An optimization problem in heat conduction. Ann. Scuola Norm. Sup. Pisa Cl. Sci. (4) 14 (1987), 355-387 (1988). Zbl 0668.49022 MR 0951225

7. Alt, H., \& CAfFARElli, L. Existence and regularity for a minimum problem with regularity. J. Reine Angew. Math. 325 (1981), 105-144. Zbl 0449.35105 MR 0618549

8. CAFFARELli, L. A. A Harnack inequality approach to the regularity of free boundaries. I. Lipschitz free boundaries are $C^{1, \alpha}$. Rev. Mat. Iberoamericana 3 (1987), 139-162. Zbl 0676.35085 MR 0990856

9. CAffarelli, L. A. A Harnack inequality approach to the regularity of free boundaries. II. Flat free boundaries are Lipschitz. Comm. Pure Appl. Math. 42 (1989), 55-78. Zbl 0676.35086 MR 0973745

10. Caffarelli, L. A., Jerison, D., \& Kenig, C. E. Global energy minimizers for free boundary problems and full regularity in three dimensions. Noncompact Problems at the Intersection of Geometry, Analysis, and Topology, Contemp. Math. 350, Amer. Math. Soc., Providence, RI (2004), 83-97. Zbl pre02166797 MR 2082392

11. Caffarelli, L. A., \& SpRUCK, J. Convexity properties of solutions to some classical variational problems. Comm. Partial Differential Equations 7 (1982), 1337-1379. Zbl 0508.49013 MR 0678504

12. Henrot, A., \& Shahgholian, H. Convexity of free boundaries with Bernoulli type boundary condition. Nonlinear Anal. 28 (1997), 815-823. Zbl 0863.35117 MR 1422187

13. Henrot, A., \& Shahgholian, H. Existence of classical solutions to a free boundary problem for the $p$-Laplace operator. I. The exterior convex case. J. Reine Angew. Math. 521 (2000), 85-97. Zbl 0955.35078 MR 1752296

14. Henrot, A., \& Shahgholian, H. Existence of classical solutions to a free boundary problem for the $p$-Laplace operator. II. The interior convex case. Indiana Univ. Math. J. 49 (2000), 311-323. Zbl 0977.35148 MR 1777029

15. Kawohl, B. Rearrangements and Convexity of Level Sets in PDE. Lecture Notes in Math. 1150, Springer, Berlin (1985). Zbl 0593.35002 MR 0810619

16. Kinderlehrer, D., \& Nirenberg, L. Regularity in free boundary problems. Ann. Scuola Norm. Sup. Pisa (4) 4 (1977), 373-391. Zbl 0352.35023 MR 0440187

17. Lavrent'ev, M. A. Variational Methods for Boundary Value Problems for Systems of Elliptic Equations. Noordhoff (1963). Zbl 0121.06701 MR 0150435

18. Lieb, E. H., \& Loss, M. Analysis. 2nd ed., Grad. Stud. Math. 14, Amer. Math. Soc., Providence, RI (2001). Zbl 0966.26002

19. OliveirA, K., \& TeiXeira, E. V. An optimization problem with free boundary governed by a degenerate quasilinear operator. Differential Integral Equations 19 (2006), 1061-1080. MR 2262097

20. Philippin, G. A. On a free boundary problem in electrostatics. Math. Methods Appl. Sci. 12 (1990), 387-392. Zbl 0712.35031 MR 1053060

21. ReICHEL, W. Radial symmetry for elliptic boundary-value problems on exterior domains. Arch. Ration. Mech. Anal. 137 (1997), 381-394. Zbl 0891.35006 |MR 1463801

22. Teixeira, E. V. A variational treatment for elliptic equations of the flame propagation type: Regularity of the free boundary. Preprint.

23. TeIXeIRA, E. V. The nonlinear optimization problem in heat conduction. Calc. Var. Partial Differential Equations 24 (2005), 21-46. Zbl 1081.49001 MR 2157849 